\title{
Plastic-Syringe Induced Silicone Contamination in Organic Photovoltaic Fabrication: Implications for Small-Volume Additives
}

\author{
John A. Carr, ${ }^{\dagger}$ Kanwar S. Nalwa, ${ }^{\ddagger}$ Rakesh Mahadevapuram, ${ }^{\ddagger}$ Yuqing Chen, ${ }^{\dagger}$ James Anderegg, ${ }^{\S}$ \\ and Sumit Chaudhary, $*,+, \neq$
}

${ }^{\dagger}$ Department of Electrical and Computer Engineering, ${ }^{\ddagger}$ Department of Materials Science Engineering, and ${ }^{\S}$ Ames Laboratory, U.S. Department of Energy, Iowa State University, Ames, Iowa 50011, United States

Supporting Information

ABSTRACT: Herein, the implications of silicone contamination found in solution-processed conjugated polymer solar cells are explored. Similar to a previous work based on molecular cells, we find this contamination as a result of the use of plastic syringes during fabrication. However, in contrast to the molecular case, we find that glass-syringe

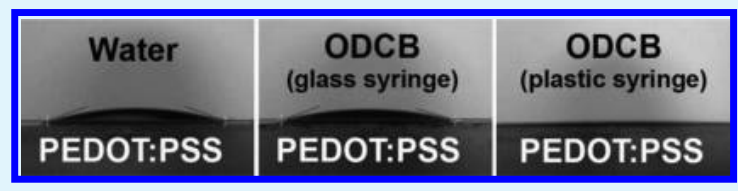
fabricated devices give superior performance than plastic-syringe fabricated devices in poly(3-hexylthiophene)-based cells. We find that the unintentional silicone addition alters the solution's wettability, which translates to a thinner, less absorbent film on spinning. With many groups studying the effects of small-volume additives, this work should be closely considered as many of these additives may also directly alter the solutions' wettability, or the amount of silicone dissolved off the plastic syringes, or both. Thereby, film thickness, which generally is not reported in detail, can vary significantly from device to device.

KEYWORDS: organic, photovoltaic, solar, polymer, silicone, plastic, PDMS, P3HT

\section{INTRODUCTION}

In the global search for clean and sustainable energy sources, organic photovoltaics (OPVs) have recently gained much attention. Facets such as solution processability, light weight, low cost, and the potential for roll-to-roll production make OPVs an advantageous option for the realization of greenpower generation. Popular modern-day organic solar cells are fabricated in what is known as the dispersed bulk-heterojunction $(\mathrm{BHJ})$ architecture, formed from blends of conjugated polymers or small molecules with fullerene derivatives. ${ }^{1}$ Since its introduction in the mid-1990s, many groups have suggested numerous methods for increasing $\mathrm{BHJ}$ power conversion efficiency (PCE). Among these are small-volume additives, in which a macromolecule or solvent are mixed with the $\mathrm{BHJ}$ blend at percentages typically less than $10 \% .^{2-10}$ Most of these reports cite an improved morphology as the reason behind performance improvement, whether it is increased mobility from larger grain sizes, or increased exciton dissociation from smaller grain sizes. What is generally overlooked is how an additive changes the wetting of the $\mathrm{BHJ}$ blend solution on the substrate surface. As we show in this work, additive-induced differential wetting can lead to a notable change in film thickness, which alone can significantly affect light absorption and photocurrent generation. Moreover, films of different thicknesses dry at different rates, which in turn can affect the internal $\mathrm{BHJ}$ morphology and other device parameters. Thus, an additive-induced differential wetting can significantly affect device performance, without playing a direct role in altering morphology of the bulk $\mathrm{BHJ}$ film. We showcase the above using silicone as a small-volume additive, which is unintentionally introduced by plastic-syringes widely used in OPV fabrication.
Recently, a work by Graham et al. showed that silicone contamination, induced during fabrication by the use of plastic syringes, can act as a small-volume additive. ${ }^{2}$ With further quantification, Graham et al. showed that a deliberate 0.05 to $1.0 \mathrm{mg} / \mathrm{mL}$ addition of polydimethylsiloxane (PDMS) to molecular based OPV solution leads to higher device efficiencies. ${ }^{2}$ The authors attributed the enhancement to a more favorable morphology, with silicone containing devices showing smaller feature sizes. In two related works, Hashimoto's group deliberately introduced poly(dimethylsiloxane)-block-poly(methyl methacrylate) (PDMS$b$-PMMA) to a poly(3-hexylthiophene):[6,6]-phenyl-C61butyric acid methyl ester (P3HT:PCBM) based system. ${ }^{11,12}$ The authors found that PDMS- $b$-PMMA aggregates near the surface and acts as a self-organizing passivation layer, which can suppress recombination at the organic/metal interface. ${ }^{12}$ Similar to the molecular case, the authors also acknowledged silicone compounds similar to PDMS- $b$-PMMA can be introduced when using disposable syringes; however, it was not speculated how these compounds might affect the performance of polymer based $\mathrm{BHJ}$ cells.

In this report, we extend these PDMS studies to include the effects of plastic-syringe induced silicone contamination on a P3HT:PCBM based BHJ OPV system. As with the molecular case, it is demonstrated that the introduction of silicone gives finer film morphology; however, this morphological change does not directly induce any performance enhancement. Further, as with the PDMS- $b$-PMMA case, we find the silicone

Received: January 29, 2012

Accepted: May 15, 2012

Published: May 15, 2012 


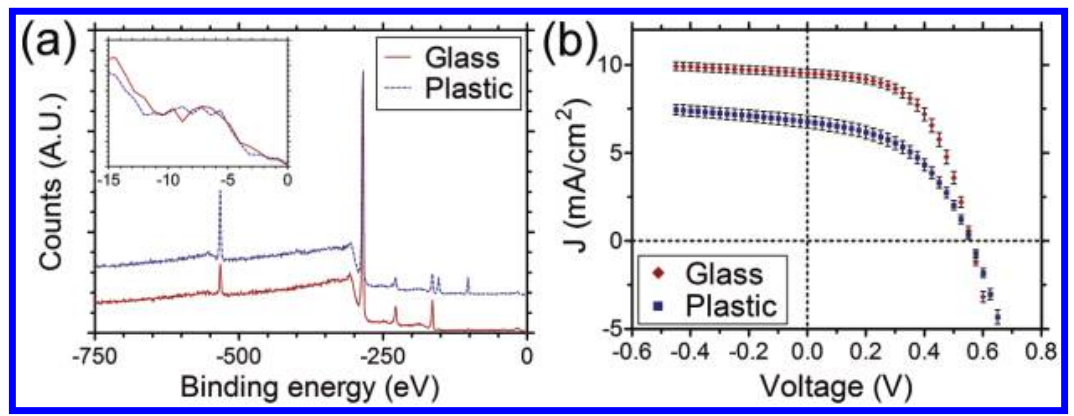

Figure 1. (a) XPS binding energy spectra for both devices (the plastic device is shifted upward for clarity). The inset shows the lower binding energies near the P3HT HOMO (ca. $4.9 \mathrm{eV}$ ). (b) $J-V$ characteristics under illumination for both devices (glass, 19 devices averaged; plastic, 25 devices averaged).

aggregates near the surface; however, this interfacial layer also does not directly lead to any performance improvement. Quite the opposite, we find silicone-deficient, glass-syringe fabricated devices produce $32.50 \pm 8.00 \%$ (mean \pm standard deviation) better cells when compared to silicone-rich, plastic-syringe fabricated devices of the same recipe. We show that the silicone contamination increases the hydrophobicity of the blend solution, altering the wettability of plastic-syringe handled solution on poly(ethylenedioxythiophene): poly(styrenesulfonic acid) (PEDOT:PSS), which generates a thinner, faster drying, and less absorbent film upon spincoating. The decrease in absorption lowers the short circuit current $\left(J_{\text {sc }}\right)$, and the faster drying time increases the defect density, decrease the shunt resistance and, thereby, lowers the fill factor (FF). These wettability-induced changes only appear in $\mathrm{BHJ}$ films spin-coated at low spin-coating speeds (600 rpm). Higher spin-coating speeds ( 800 and $1000 \mathrm{rpm}$ ) overcome the wettability effect and silicone contaminated devices perform similar to their noncontaminated counterparts.

\section{RESULTS AND DISCUSSION}

Within the research community, spin-coating is a preferred OPV fabrication technique as it is simple, consistent, and allows for small-scale production. Although spin-casting details may vary from group to group, this technique generally requires the use of syringes to transfer and filter polymer solutions. For ease of use and convenience, many opt to use disposable, plastic syringes. However, these commercially available syringes are typically lubricated with medicated silicone, ${ }^{13}$ which is easily dissolved, and introduced to the OPV system, by commonly used organic solvents. Here, we investigate this issue in its strictest sense: by directly comparing 1:1 P3HT:PCBM BHJ OPVs fabricated from plastic-syringe handled solution to that of OPVs fabricated from glass-syringe handled solution. Plastic (i.e., plastic-syringe handled solution) and glass (i.e., glasssyringe handled solution) devices were derived from three spin speeds, (i) $600 \mathrm{rpm}$, (ii) $800 \mathrm{rpm}$, and (iii) $1000 \mathrm{rpm}$, and shared the same structure: glass/indium-doped tin oxide (ITO)/ PEDOT:PSS/P3HT:PCBM/Aluminum (Al). Details of the fabrication process are provided in the Supporting Information.

We first investigated OPV devices with active layer films spun at $600 \mathrm{rpm}$ for 60s. The X-ray photoelectron spectroscopy (XPS) binding-energy spectra is presented in Figure 1a. XPS confirms plastic devices contain a silicone contamination, while glass devices are silicone free (Figure 1a). Two silicon ( $\mathrm{Si}$ ) peaks (found at 152 and $101 \mathrm{eV}$ ) are slightly shifted from their expected values ( $\mathrm{Si} 2 \mathrm{~s}$ at $149.7 \mathrm{eV}$ and $\mathrm{Si} 2 \mathrm{p}$ at $99.42 \mathrm{eV}){ }^{14}$ indicating silicon is bonding with a second element. This, coupled with the plastic devices' increased, and also shifted, oxygen peak (near $\mathrm{O} 1 \mathrm{~s}$ at $543.1 \mathrm{eV})^{14}$ shows strong evidence that silicone, and not bare silicon, is present. Using argon etching, we find the silicone contamination tends to aggregate near the surface, with ca. $8.00 \pm 1.00 \%$ atomic concentration of silicone within the films' top 1 to $2 \mathrm{~nm}$, ca. $1 \%$ from 2 to $10 \mathrm{~nm}$, and nearly $0 \%$ throughout the remaining bulk (see SFigure 1 in the Supporting Information for details). This is a result of the lower surface energy of PDMS, ${ }^{15,16}$ and mimics the case of P3HT:PCBM:PDMS- $b$-PMMA based cells. ${ }^{12}$ The inset of Figure 1a shows valence band measurements of both devices, with no difference between the devices' highest occupied molecular orbital (HOMO; ca. $4.9 \mathrm{eV}$ ) found.

Figure $1 \mathrm{~b}$ shows the current-votlage $(J-V)$ characteristics of both devices. The open circuit voltage $\left(V_{\mathrm{oc}}\right)$ showed no significant variation ( $\mathrm{p}$ value $\gg 0.05$; unpaired $t$ test) between plastic $(0.561 \pm 0.02 \mathrm{~V} ; n=19)$ and glass $(0.562 \pm 0.01 \mathrm{~V} ; n=$ $25)$ devices. The fill factor $(\mathrm{FF})$ and short circuit current $\left(\mathrm{J}_{s c}\right)$, however, varied significantly $(\mathrm{p}$ value $<0.01)$; with $\mathrm{FF}_{\text {plastic }}=$ $51.10 \pm 10.2 \%, \mathrm{FF}_{\text {glass }}=59.50 \pm 3.4 \%, J_{\text {sc }}$ plastic $=6.77 \pm 0.76$ $\mathrm{mA} \mathrm{cm}{ }^{-2}$, and $J_{\mathrm{sc}}$ glass $=9.50 \pm 0.82 \mathrm{~mA} \mathrm{~cm}^{-2}$.

After detailed analysis, we find that glass-syringe fabrication generates a ca. $24.70 \%$ thicker active-layer film. Figure 2a shows the five-number summary (sample minimum, Q1, Q2, Q3, and sample maximum) for the atomic force microscopy (AFM)

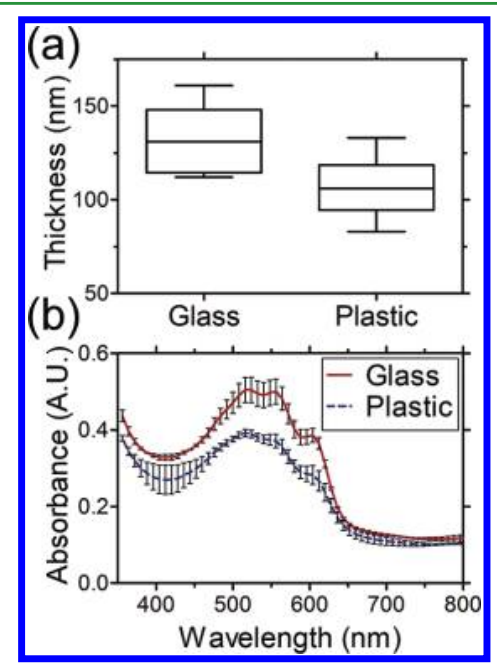

Figure 2. (a) Five-number summary of active-layer thicknesses for films spun at $600 \mathrm{rpm}$ for $60 \mathrm{~s}$. (b) Absorbance spectra of glass and plastic devices spun at $600 \mathrm{rpm}$ for $60 \mathrm{~s}$. 


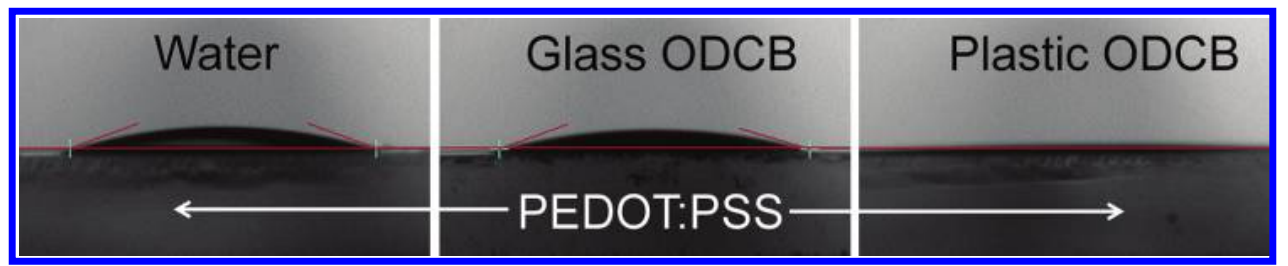

Figure 3. Image of contact angle measurement for water, glass-syringe handled ODCB, and plastic-syringe handled ODCB on PEDOT:PSS.

thickness measurement of 10 films (see SFigure 2 in the Supporting Information for measurement details). Glasssyringe fabrication produces a significantly ( $p$ value $<0.01$ ) thicker film $(133.6 \pm 17.6 \mathrm{~nm})$ than the plastic-syringe fabrication $(107.3 \pm 15.4 \mathrm{~nm})$. As expected, the glass devices' thicker active-layer film leads to an enhancement in absorbance (Figure 2b), which translates to the higher photogenerated current shown in Figure $1 \mathrm{~b}$.

During the fabrication of these devices, we noticed a large difference in the solutions' wettability on the glass/ITO/ PEDOT:PSS substrate. Thus, we next look at the spreading of similar sized ODCB droplets dispersed by both glass and plastic syringes. Contact angle measurements were carried out on an Image Analysis Ganiometer (Rame-Hart, Inc. model 100-22(v)) using a static sessile drop method. $0.5 \mu \mathrm{L}$ droplets of (i) water, (ii) glass-syringe filtered ODCB, and (iii) plastic-syringe handled ODCB were placed on a substrate and imaged. PEDOT:PSS, microscope glass, and bare ITO substrates were tested. Figure 3 shows sample images from one such experiment with PEDOT:PSS as the substrate. Water and glass solutions showed similar spreading with contact angles of $16.9 \pm 1^{\circ}$ and $17 \pm 2^{\circ}$, respectively. The measured contact angle of water on PEDOT:PSS is similar to value reported in literature. ${ }^{17}$ The plastic solution, however, showed perfect spreading with no measurable angle. These differences are further exaggerated on glass and ITO substrates (see SFigure 3 in the Supporting Information). These results reveal that the introduction of silicone generates a more hydrophobic solution, which spreads much more easily and generates a thinner film on spinning.

Coupled with a change in film thickness, is a change in growth rate (see SFigure 4 in the Supporting Information for dry times). The increased performance of glass devices has thus far been imputed to an increase in $J_{\text {sc }}$ stemming from an increase in optical density. However, the effects of changes in crystallization kinetics have not yet been accounted for. Drying a film more slowly can have positive effects on OPV morphology. These effects can include: higher exciton generation, higher carrier mobility, lower defect densities, as well as higher exciton dissociation efficiency. ${ }^{18-21}$ Further, in the case of PDMS- $b$-PMMA, it was shown that upon adding an excessive amount of PDMS a thick electron transport barrier was formed, and performance was degraded. ${ }^{12}$ Thus, in effort to explicate any further variations caused by the differential thickness, we next study the surface morphology, deep defect profiles, and dark current.

Atomic force microscopy was used to study the surface morphology of both films (see SFigure 5a-d in the Supporting Information). The plastic fabrication process produces slightly smoother films (plastic mean roughness $\left(R_{\mathrm{a}}\right)=6.35 \pm 0.78 \mathrm{~nm}$; glass $R_{\mathrm{a}}=8.0 \pm 0.3 \mathrm{~nm}$ ) with slightly smaller feature sizes. This corroborates with the molecular PV based work. ${ }^{2}$ Here, we first theorized that the augmented thickness and drying times of the glass devices encouraged crystallinity and larger grain formation. However, the Raman shift for plastic and glass devices (see SFigure 6 in the Supporting Information) showed no difference, signifying no significant difference in bulk crystallinity despite the difference in growth rate. ${ }^{21,22}$ This, coupled with the finding that the silicone aggregates in the top $2 \mathrm{~nm}$ of the film, indicates that the morphological change could be only a surface phenomenon.

However, the above would not explain the lowered FF in plastic devices. Thus, we turn to the density of deep defects (see SFigure 7 in the Supporting Information). The trap profiles were calculated using a capacitance method detailed elsewhere. $^{23,24}$ In contrast to the Raman data, the defect profiles show a slight shift in the magnitude of trap states, which is a direct result of the decreased growth rate in plastic devices. We note that, albeit statistically significant, this shift is relatively small. Nevertheless, a slight increase in traps states can lead to a small reduction in FF via increased recombination. $^{21,25}$ Further, the decreased dry times can allow for more shunt paths to percolate from anode to cathode and, thereby, a smaller shunt resistance in the plastic devices. Figure $4 \mathrm{a}$ shows the logarithm of dark current versus voltage for

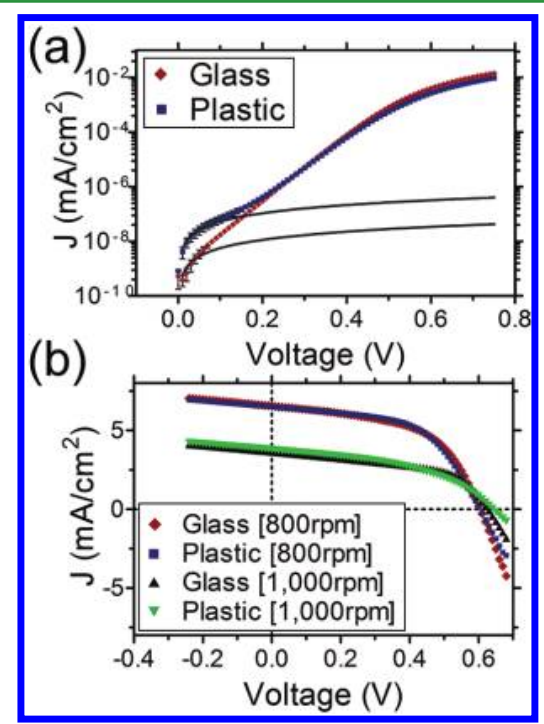

Figure 4. (a) Logarithm of dark current versus voltage for both devices. (b) $J-V$ characteristics for plastic- and glass-syringe fabricated devices spun at $800 \mathrm{rpm}$ (top two curves) and $1000 \mathrm{rpm}$ (bottom two curves). Error bars removed for added clarity.

both devices studied in this report. Here, we employ a single diode circuit model that is detailed elsewhere. ${ }^{26-28}$ In this model six equations are used to fit the data in three different voltage regions: (i) $V \lesssim 0.2$, (ii) $0.2 \lesssim V \lesssim 0.5$, and (iii) $V \gtrsim$ 0.5 . Figure 4 a shows a difference between the glass and plastic devices is in the low voltage region, revealing the difference in the shunt resistance $\left(R_{\mathrm{sh}}\right.$, plastic $\approx 2 \times 10^{7} \Omega ; R_{\mathrm{sh}}$, glass $\approx 2 \times$ $10^{8} \Omega$ ). This decay in shunt resistance in plastic devices also 
leads to a reduction in $\mathrm{FF}^{29}$ Thus, some morphological changes appear to be present throughout the bulk.

To ensure these minor morphological changes do indeed stem from the altered dry times, and not directly from the presence of silicone itself, we subsequently increase the casting speed to fabricate glass and plastic films of similar thicknesses. This isolates the thickness/growth-rate effect from more direct augmentations caused by silicone. 800 and 1,000 rpm are tested in an effort to negate the wettability effect. Figure $4 \mathrm{~b}$ displays the $J-V$ characteristics under illumination for these films. In both cases, the glass and plastic devices performed equally, with no significant difference ( $\mathrm{p}$ value $\gg 0.05$ ) in any performance parameter found. AFM measurements confirm similar film thicknesses for the $800 \mathrm{rpm}$ glass and plastic devices $\left(T_{\text {glass }}=\right.$ $115.0 \pm 6.8 \mathrm{~nm} ; T_{\text {plastic }}=107.0 \mathrm{~nm} \pm 7.3 \mathrm{~nm} ; \mathrm{p}$ value $\left.>0.05\right)$, as well as the $1000 \mathrm{rpm}$ devices $\left(T_{\text {glass }}=87.2 \pm 5 \mathrm{~nm}\right.$; $\mathrm{T}_{\text {plastic }}=84.8$ $\pm 5.8 \mathrm{~nm} ; \mathrm{p}$ value $\gg 0.05)$. This shows the silicone contamination is not affecting device parameters when the thickness is forced to be similar. Further, this data highlights that the wetting effect is dependent on the spin-casting recipe and, more generally, that the inhibition or usefulness of an additive can be dependent on the fabrication environment.

It is worth commenting on the variability among these devices. Here, we have controlled the solutions' exposure to silicone by allowing the solution to sit in the syringe for $2 \mathrm{~min}$. In spite of this controlled exposure, we still noticed a larger variation in the PCE of plastic-fabricated devices. Glass devices showed a median PCE of $3.20 \%$ with a standard deviation of $0.40 \%$, whereas plastic devices presented a median of $2.30 \%$ with a standard deviation of $0.70 \%$. Intuitively, this deviation difference can be attributed to variations in the amount of silicone dissolved from the syringe. Thus, we expect those groups not carefully controlling exposure may see even larger device to device deviation. Further, we expect large variations from lab to lab can also, in part, be imputed to differences in silicone contamination. Lastly, we note that needle tips can also be "siliconized" 30 and leach silicone into the system. Here, we have used these lubricated tips, but only occasionally found small traces of Si in XPS.

\section{CONCLUSION}

In summary, we confirm the previously reported silicone-based contamination in plastic-syringe fabricated OPVs and extend to characterize its implications as a small volume additive in conjugated polymer based OPVs. We investigate this issue by directly comparing devices fabricated from glass- and plasticsyringes in a P3HT:PCBM BHJ system. We find slow-spun glass fabricated devices give superior PCEs than that of plastic fabricated devices of the same recipe. We attribute the degradation of the plastic devices' performance to a thinner, less absorbent active layer film. The thinned film stems from the addition of silicone to the active-layer blend which alters the solution's wettability on the substrate. Casting at faster speeds produced plastic and glass devices with similar PCEs, showing altered thickness is the dominate facet affecting the plastic cells. It remains an open question as to how a plastic-syringe induced silicone contamination will interact with and affect the next generation, high efficiency polymers (e.g., poly[[9-(1-octylnonyl)-9H-carbazole-2,7-diyl]-2,5-thiophenediyl-2,1,3-benzothiadiazole-4,7-diyl-2,5-thiophenediyl]). Further, using this report as an initial vehicle, other groups pursuing small-volume additives should also consider the possibility of a changed wettability resulting directly from the additive itself or indirectly by altering the amount of silicone dissolved by the active blend solution.

\section{ASSOCIATED CONTENT}

\section{Supporting Information}

Associated content, including (i) Experimental details, (ii) XPS etching, (iii) AFM thickness measurements, (iv) contact angle measurements, (v) dry times, (vi) AFM roughness and phase diagrams, (vii) Raman spectroscopy, (viii) capacitance profiling data, (ix) Voc versus light intensity, and (x) external quantum efficiency. This material is available free of charge via the Internet at http://pubs.acs.org.

\section{AUTHOR INFORMATION}

\section{Corresponding Author}

*Address: 2124 Coover Hall ,Iowa State University, Ames, IA 50011, USA. Phone: 515.294.0606. Fax: 515.294.1152. E-mail: sumitc@iastate.edu.

\section{Notes}

The authors declare no competing financial interest.

\section{ACKNOWLEDGMENTS}

We thank National Science Foundation (ECCS - 1055930) for financial support. XPS studies were carried out at the Ames Laboratory, which is operated for the U.S. Department of Energy by Iowa State University under Contract No. DEAC02-07CH11358. These studies were supported by the U.S. Department of Energy, Office of Basic Energy Sciences, Division of Materials Sciences and Engineering.

\section{REFERENCES}

(1) Yu, G.; Gao, J.; Hummelen, J. C.; Wudl, F.; Heeger, A. J. Science 1995, 270 (5243), 1789-1791.

(2) Graham, K. R.; Mei, J.; Stalder, R.; Shim, J. W.; Cheun, H.; Steffy, F.; So, F.; Kippelen, B.; Reynolds, J. R. ACS Appl. Mater. Interfaces 2011, 3 (4), 1210-5.

(3) Peet, J.; Soci, C.; Coffin, R.; Nguyen, T.; Mikhailovsky, A.; Moses, D.; Bazan, G. C. Appl. Phys. Lett. 2006, 89 (25), 252105-252105-3.

(4) Zhang, F.; Jespersen, K. G.; Björström, C.; Svensson, M.; Andersson, M. R.; Sundström, V.; Magnusson, K.; Moons, E.; Yartsev, A.; Inganäs, O. Adv. Funct. Mater. 2006, 16 (5), 667-674.

(5) Peet, J.; Kim, J. Y.; Coates, N. E.; Ma, W. L.; Moses, D.; Heeger, A. J.; Bazan, G. C. Nat. Mater. 2007, 6 (7), 497-500.

(6) Lee, J. K.; Ma, W. L.; Brabec, C. J.; Yuen, J.; Moon, J. S.; Kim, J. Y.; Lee, K.; Bazan, G. C.; Heeger, A. J. J. Am. Chem. Soc. 2008, 130 (11), 3619-3623.

(7) Yao, Y.; Hou, J. H.; Xu, Z.; Li, G.; Yang, Y. Adv. Funct. Mater. 2008, 18 (12), 1783-1789.

(8) Hoven, C. V.; Dang, X. D.; Coffin, R. C.; Peet, J.; Nguyen, T. Q.; Bazan, G. C. Adv. Mater. 2010, 22 (8), E63-+.

(9) Chang, L.; Lademann, H. W. A.; Bonekamp, J.-B.; Meerholz, K.; Moulé, A. J. Adv. Funct. Mater. 2011, 21 (10), 1779-1787.

(10) Chu, T. Y.; Alem, S.; Tsang, S. W.; Tse, S. C.; Wakim, S.; Lu, J.; Dennler, G.; Waller, D.; Gaudiana, R.; Tao, Y. Appl. Phy. Lett. 2011, 98, 253301.

(11) Nishizawa, T.; Lim, H. K.; Tajima, K.; Hashimoto, K. Chem. Commun. 2009, 18, 2469-2471.

(12) Yamakawa, S.; Tajima, K.; Hashimoto, K. Org. Electron. 2009, 10 (3), 511-514.

(13) Syringes, See www.fishersci.com: BD* Disposable Syringes for example.

(14) Thompson, A. C.; Vaughan, D. X-ray Data Booklet; Center for X-ray Optics and Advanced Light Source, Lawrence Berkeley National Laboratory: Berkeley, CA, 2001. 
(15) Wang, X.; Ederth, T.; Inganäs, O. Langmuir 2006, 22 (22), 9287-9294.

(16) Mata, A.; Fleischman, A. J.; Roy, S. Biomed. Microdevices 2005, 7 (4), 281-293.

(17) Ouyang, J.; Xu, Q.; Chu, C. W.; Yang, Y.; Li, G.; Shinar, J. Polymer 2004, 45 (25), 8443-8450.

(18) Li, G.; Shrotriya, V.; Yao, Y.; Yang, Y. J. Appl. Phys. 2005, 98, 4.

(19) Shrotriya, V.; Yao, Y.; Li, G.; Yang, Y. Appl. Phys. Lett. 2006, 89, 6.

(20) Li, G.; Yao, Y.; Yang, H.; Shrotriya, V.; Yang, G.; Yang, Y. Adv. Funct. Mater. 2007, 17 (10), 1636-1644.

(21) Nalwa, K. S.; Mahadevapuram, R. C.; Chaudhary, S. Appl. Phys. Lett. 2011, 98, 093306.

(22) Casado, J.; Hicks, R.; Hernandez, V.; Myles, D.; Delgado, M. C. R.; Navarrete, J. T. L. J. Chem. Phys. 2003, 118, 1912.

(23) Walter, T.; Herberholz, R.; Muller, C.; Schock, H. J. Appl. Phys. 1996, 80 (8), 4411-4420.

(24) Boix, P. P.; Garcia-Belmonte, G.; Munecas, U.; Neophytou, M.; Waldauf, C.; Pacios, R. Appl. Phys. Lett. 2009, 95 (23), 233302-3.

(25) Günes, S.; Neugebauer, H.; Sariciftci, N. S. Chem. Rev. 2007, 107 (4), 1324-1338.

(26) Nolasco, J.; Cabre, R.; Ferré-Borrull, J.; Marsal, L.; Estrada, M.; Pallares, J. J. Appl. Phys. 2010, 107 (4), 044505-044505-4.

(27) Pallarès, J.; Cabré, R.; Marsal, L.; Schropp, R. J. Appl. Phys. 2006, $100,084513$.

(28) Nolasco, J.; Cabre, R.; Marsal, L.; Pacios, R.; Waldauf, C.; Neophytou, M.; Palomares, E.; Pallares, J. Conduction Mechanisms of P3HT: PCBM Solar Cell. In Spanish Conference on Electron Devices 2009; Santiago de Compostela, Spain, Feb 11-13, 2009 IEEE: New York, 2009; pp 398-401.

(29) Kim, M. S.; Kim, B. G.; Kim, J. ACS Appl. Mater. Interfaces 2009, 1 (6), 1264-1269.

(30) Needles, See www.fishersci.com: BD* PrecisionGlide* Needles for example. 